\title{
MPEG2 video parameter and no reference PSNR estimation
}

\section{Li, Huiying; Forchhammer, Søren}

\section{Published in:}

Picture Coding Symposium, 2009. PCS 2009

Link to article, DOI:

10.1109/PCS.2009.5167426

Publication date:

2009

Document Version

Publisher's PDF, also known as Version of record

Link back to DTU Orbit

Citation (APA):

$\mathrm{Li}, \mathrm{H}$., \& Forchhammer, S. (2009). MPEG2 video parameter and no reference PSNR estimation. In Picture Coding Symposium, 2009. PCS 2009 (pp. 1-4). IEEE. https://doi.org/10.1109/PCS.2009.5167426

\section{General rights}

Copyright and moral rights for the publications made accessible in the public portal are retained by the authors and/or other copyright owners and it is a condition of accessing publications that users recognise and abide by the legal requirements associated with these rights.

- Users may download and print one copy of any publication from the public portal for the purpose of private study or research.

- You may not further distribute the material or use it for any profit-making activity or commercial gain

- You may freely distribute the URL identifying the publication in the public portal

If you believe that this document breaches copyright please contact us providing details, and we will remove access to the work immediately and investigate your claim 


\title{
MPEG2 VIDEO PARAMETER AND NO REFERENCE PSNR ESTIMATION
}

\author{
Huiying Li and Søren Forchhammer \\ Technical University of Denmark \\ DTU Fotonik \\ B. 343, 2800 Lyngby, Denmark
}

\begin{abstract}
MPEG coded video may be processed for quality assessment or postprocessed to reduce coding artifacts or transcoded. Utilizing information about the MPEG stream may be useful for these tasks. This paper deals with estimating MPEG parameter information from the decoded video stream without access to the MPEG stream. This may be used in systems and applications where the coded stream is not accessible. Detection of MPEG I-frames and DCT (Discrete Cosine Transform) block size is presented. For the I-frames, the quantization parameters are estimated. Combining these with statistics of the reconstructed DCT coefficients, the PSNR is estimated from the decoded video without reference images. Tests on decoded fixed rate MPEG2 sequences demonstrate perfect detection rates and good performance of the PSNR estimation.
\end{abstract}

Index Terms - MPEG, No reference PSNR, quantization, parameter estimation, I-frame detection

\section{INTRODUCTION}

MPEG video coding is based on coding block based DCT (Discrete Cosine Transform) coefficients for I (Intra) frames. For the other frames, motion compensation is applied prior to the DCT. The distortion is determined by the quantization applied to the DCT coefficients. Decoded MPEG video may be transcoded for storage or further transmission or subjected to postprocessing to attenuate the coding artifacts and thereby increase quality. In some applications it may be desirable to assess the quality of the video or analyze it for forensic purposes. For all these tasks the parameters of the MPEG stream can provide useful information [1][2].

The parameters can be extracted from the coded stream, e.g. at the time of decoding. In many cases, the coded stream is not accessible, or from an architectural point of view, it is desirable not to access it. In some cases, a (non-encrypted) stream is not accessible out of the decoder or out of a third party (hardware) decoder. In other cases, it may be desirable just to use existing hardware/software decoder(s). Especially if we consider a set-up where the decoded video can come from a range of external decoders, it may be desirable to base the processing or analysis solely on the decoded signal. Improved performance may in this case be achieved by estimating some of the parameters used when coding the MPEG video, but based on the decoded video. As an extra challenge the output of the decoder may have been processed eg. by scaling before it is accessible.

As examples we consider estimating MPEG2 quality and extracting MPEG2 parameters. This may be used for aiding postprocessing. The MPEG video distortion and artifacts come from the DCT domain quantization. The distortion and strength of artifacts are correlated with the values of the quantization step sizes, which are given by the MPEG2 parameters quantization scale $\left(Q_{S}\right)$ and

This work was supported by the Danish Strategic Research Council. quantization matrix $\left(Q_{M}\right)$. The MPEG2 frame types (I frame or motion compensated) possess different artifacts and identifying I frames is also beneficial when transcoding. The DCT block size and DCT block boundary positions may be used to localize MPEG blocking artifacts in a deblocking filter. The MPEG2 block size is $8 \times 8$, but the video data may have been rescaled after decoding.

In this paper we focus on some elements of MPEG analysis namely the important MPEG parameters: DCT block size and position detection (or validation), I-frame detection and for the I frames, estimation of the quantization step size. Furthermore we shall utilize this information to estimate the PSNR of the I frames. A likelihood based measure is introduced as part of the detection and estimation as well as validating that the stream has been MPEG2 coded.

Methods for (decoder side) no reference PSNR estimation, i.e. without access to the original video, have been presented based on MPEG parameters extracted from the streams [1]. In [3] the analysis is just based on the decoded stream. For the I frames, estimation of the quantization parameters and PSNR was presented by modelling the distribution of the DCT coefficients. Expressions and results were provided for fixed quantizer video. We extend this to fixed rate and thereby variable quantizer values and furthermore introduce a measure to validate the analysis.

The rest of the paper is organized as follows: MPEG2 decoding is briefly described in Sect. 2. Section 3 introduces the MPEG2 parameter estimation based on the decoded video: block size and position estimation, quantization step size estimation, and I frame detection. Section 4 describes the use of the recovered parameters for PSNR estimation on I frames. Experimental results are given in Sect. 5 .

\section{MPEG2 DECODING PROCESS}

To provide the background and notation for the analysis, the decoding of MPEG2 I frames is briefly described. In MPEG2, the basic processing unit is the $16 \times 16$ pixel (luminance) Macro Block (MB), which is further divided into four $8 \times 8$ blocks, which are transformed using the DCT. The transformed coefficients are quantized, which is locally controlled by one quantizer scale value, $Q_{S}$, per MB.

The main processes of the MPEG2 decoder includes Variable Length Decoding (VLD), Inverse Quantization and Inverse DCT transformation as depicted on Fig.1. The output of the VLD, $I_{Q}(u, v)$, is an integer value, which represents the index of the quantization interval for the DCT coefficient at $(u, v)$. Based on this the DCT coefficients $F^{\prime \prime}(u, v)$ for the reconstruction are determined. The DCT AC coefficients, i.e. $(u, v) \neq(0,0)$, are reconstructed in conformance with [4]. For the intra MB this gives

$$
\left|F^{\prime \prime}(u, v)\right|=\left\lfloor\frac{\left|I_{Q}(u, v)\right| \times Q_{M}(u, v) \times Q_{S}}{16}\right\rfloor
$$

where \lfloor\rfloor denotes the floor function and $Q_{M}(u, v)$ denote the frequency dependent quantization matrix values. The four luma DCT 


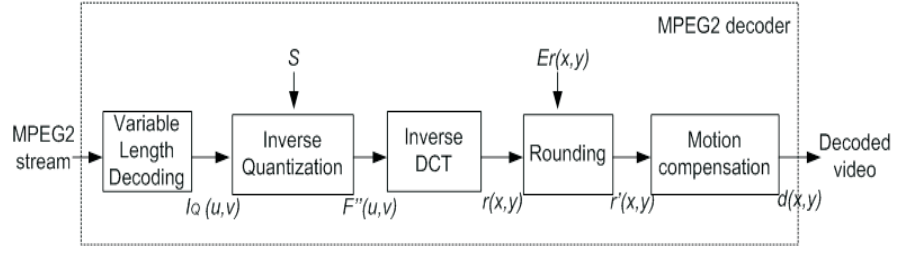

Fig. 1. The MPEG2 decoding process.

blocks in one macro-block are quantized using the same $Q_{S}$ value, but $Q_{S}$ may change from one $\mathrm{MB}$ to the next. It should be noted that $F^{\prime \prime}, I_{Q}, Q_{M}(u, v)$ and $Q_{S}$ all are integers.

After $F^{\prime \prime}(u, v)$ is reconstructed, the inverse DCT will convert $F^{\prime \prime}(u, v)$ to the inverse transformed value $r(x, y)$, which is rounded, and, if necessary clipped, to obtain integer values $r^{\prime}(x, y)$ in the range $[0,255]$. For intra blocks, $r^{\prime}(x, y)$ will be directly output as the decoded video, $d(x, y)$. For non-intra blocks $r^{\prime}(x, y)$ will further be combined with motion compensated data for the output $d(x, y)$.

\section{MPEG2 PARAMETER ESTIMATION}

MPEG2 parameters are estimated based on the decoded video $d(x, y)$. The focus is on estimating or detecting three main parameters: DCT blocksize and position, Quantization step size, and I frames. The overall process is shown in Fig. 2. The first DCT block boundary positions are estimated both horizontally and vertically. Based on that, the $8 \times 8$ block DCT is applied on each DCT block. (If the detected block size is not $8 \times 8$, the DCT blocks should first be rescaled into $8 \times 8$, then DCT transformed). Both frame and field DCT may be applied on MBs. The MB_type (frame \& field MB) can be estimated by selecting the type minimum number of the zero DCT coefficients for an MB. Thereafter, $Q_{M}$ and $Q_{S}$ estimation are applied based on the reconstructed DCT coefficients $F^{\prime}(u, v)$ (frame DCT if the MB is evaluated to be a frame MB, otherwise field DCT). The quantization step sizes (for each of the DCT coefficients) are recovered based on the estimated $Q_{M}$ and $Q_{S}$. Furthermore the MB level mismatch $\left(M_{M B}\right)$ is obtained. Frame level mismatch $\left(M_{F}\right)$ is then calculated by the frame level average of $M_{M B}$. Finally I frames are detected by $M_{F}$.

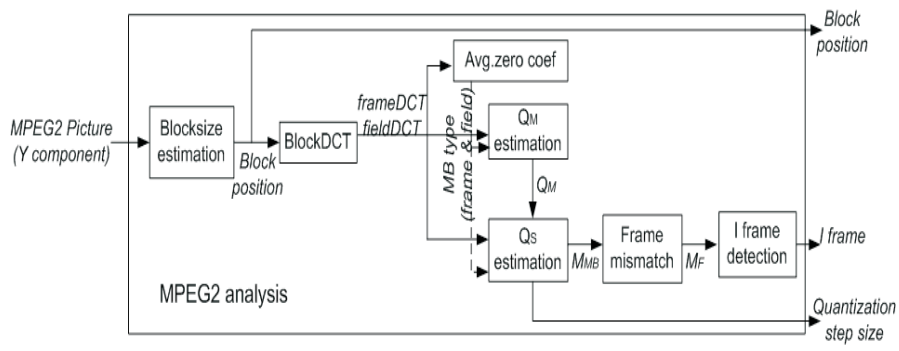

Fig. 2. MPEG2 frame level analysis.

\subsection{Blocksize estimation}

Absolute differences between adjoining pixels have been used to perform a blocking artifacts analysis [5]. Instead we calculate difference of differences and project the values on the horizontal and vertical axis (by summation). Applying a frequency analysis (using FFT) to these projected values gives clear peaks corresponding to the block structure.

\subsection{Quantization step size estimation}

For intra MBs, the quantized MPEG2 DCT coefficients can be recovered by applying an $8 \times 8$ DCT to the decoded video $d(x, y)$. Without the information of motion vectors and residues, it is intractable to recover non-intra DCT coefficients. We consider the general case where Intra/non-intra frame and MB type information is not known (or uncertain). Initially, the proposed method treats all MBs as intra. After the processing, a decision about Intra/non-intra frames is made and validated. The Intra frame quantization step size, $\Delta$, is a function of the DCT frequency $(u, v)$,

$$
\Delta(u, v)=\frac{Q_{S} \times Q_{M}(u, v)}{16}
$$

It may be necessary to estimate both $Q_{M}(u, v)$ and $Q_{S}$. The basic idea of recovering $Q_{S}$ (and $Q_{M}(u, v)$ ) on I frames is first to apply DCT on $8 \times 8$ blocks of the decoded video $r^{\prime}(x, y)$ to recover the DCT coefficients, $F^{\prime}(u, v)$, and based on these estimate $I_{Q} \times$ $Q_{S} \times Q_{M}$ (1) for each DCT coefficient $(u, v)$, where $Q_{M}$ is fixed at frame level and $Q_{S}$ at MB level. In principle $Q_{S}$ and $Q_{M}(u, v)$ are identical to the greatest common divisor (or a divisor of this) at MB and frame level, respectively [3].

This paper starts with $Q_{M}$ estimation because $Q_{M}$ is fixed for the whole frame, thus providing more statistics. If we restrict the analysis to a limited set of candidate $Q_{M}$, the problem is simplified to that of identifying (and validating) the selected candidate.

Having $Q_{M}, I_{Q}(u, v) \times Q_{S}$ may be recovered to estimate $Q_{S}$ using a greatest common divisor approach. However, it is not certain that the values of $I_{Q}(u, v) \times Q_{S}$ obtained are the correct ones due to the non-linear processing of the rounding (and clipping) after the inverse DCT transformation and the integer division in the decoder. Empirically, we have noted that the distribution of the rounding error can be approximated by a Laplace distribution. Under this assumption, the estimation of $Q_{S}$ can be expressed as a maximum likelihood problem. Taking the logarithm, this maybe expressed as finding the minimum of a sum of terms given by

$$
\min _{n}\left(\left|F^{\prime}(u, v)-n \Delta(u, v)\right|\right), n>0
$$

where the argument is the distance between the reconstructed DCT value $F^{\prime}(u, v)$ and the nearest possible reconstructed value of the MPEG2 decoder (2). Based on these ideas, practical and robust estimation schemes are presented below.

\subsection{1. $Q_{M}$ matrix estimation}

The MPEG2 default intra $Q_{M}$ is widely used in many applications. This was confirmed by analysis of some on-air MPEG2 sequences where also one more $Q_{M}$ matrix was observed. These two matrices constitute our candidate set.

As noted $Q_{M}$ is a sequence level parameter, and all $\mathrm{AC}$ coefficients of a given frequency, $(u, v)$ is quantized using the same $Q_{M}$ value. The four DCT blocks within the same MB have the same $Q_{S}$. The candidate $Q_{S}$ values are tested on the MB and the $Q_{S}$ having the smallest MB mismatch value, $M_{M B}$ is selected:

$$
\hat{Q}_{M}=Q_{M}(p): \arg \min _{p \in\left\{Q_{M}\right\}} \sum_{M B} \min _{q \in\left\{Q_{s}\right\}}\left(M_{M B}(p, q)\right)
$$

where

$$
\begin{aligned}
& M_{M B}\left(Q_{M}, Q_{s}\right) \\
= & \sum_{(u, v) \in M B} \mid \text { round }\left(\frac{F^{\prime}(u, v) \times 16}{Q_{M}(u, v) \times Q_{S}}\right)-\frac{F^{\prime}(u, v) \times 16}{Q_{M}(u, v) \times Q_{S}} \mid
\end{aligned}
$$

The distance related to the log-likelihood formulation (3) is modified by normalizing by the quantization step size to make the mismatch independent of $Q_{S}$ and $Q_{M}$, which will also favor the lower frequencies.

\subsection{2. $Q_{S}$ quantizer scale estimation}

As MPEG2 has a unique $Q_{S}$ for each MB, the decoded DCT coefficients $F^{\prime \prime}(u, v)$ within one MB will be distributed on integer multiples of the quantization step size [3] (with integer division truncation shift $S$ ) leading to the terms $\left(\left|I_{Q}\right| \times Q_{S}\right)$. For a single MB, 
$Q_{S}$ can then be estimated as the greatest common divisor (gcd) of the $\left(\left|I_{Q}\right| \times Q_{S}\right)$. The reconstructed values of $\left(\left|I_{Q}\right| \times Q_{S}\right)$ from the decoded video may be in error due to the decoder rounding error $E_{r}$, the integer division shift $S$ and other possible processing steps ( $E_{r}$ and $S$ are shown in Fig. 1). As the gcd operator is highly non-linear, where one wrong input value can lead to a completely wrong estimation, a more robust algorithm was developed. It is known that $0 \leq S<1$, and we assume that the probability distribution of the rounding error in DCT domain $E_{r}(u, v)$ may be approximated by a Laplace distribution. Integer upper $\left(\left(\left|I_{Q}\right| \times Q_{S}\right)_{\text {up }}\right.$ and lower $\left.\left(\left|I_{Q}\right| \times Q_{S}\right)_{\text {down }}\right)$ bounds are defined as follows:

$$
\left\lceil\frac{16\left(F^{\prime}-E_{\max }\right)}{Q M}\right\rceil \leq\left|I_{Q}\right| \times Q_{S} \leq\left\lceil\frac{16\left(F^{\prime}+E_{\max }\right)}{Q M}\right\rceil
$$

where $E_{\max }$ was determined by experimentally fitting a Laplace distribution to the $\operatorname{Er}(u, v)$ for each DCT coefficient, and selecting $E_{\max }$ at the $99 \%$ level of the cumulative density function.

Several observations are used in the estimation algorithm below:

1) The $\operatorname{set}\left\{Q_{S}\right\}$ of all potential values of $Q_{S}$ are given by the MPEG2 $Q_{S}$ table, which is defined by MPEG2 [4].

2) For a single $\mathrm{MB}$, the $Q_{S}$ upper bound, $Q_{S}^{u p}$, can be obtained by $\min \left(\left(I_{Q} \times Q_{S}\right)_{u p}\right)$ (min is over the frequencies $(u, v)$ for all the non-zero DCT coefficients).

3) MPEG2 has a bias towards maintaining the same $Q_{S}$ value as the previous MB. Therefore the previous $Q_{S}$ can be used for estimation.

\section{The $Q_{S}$ estimation algorithm For each MB do}

1. For all the AC DCT values, $F^{\prime}(u, v)(u, v) \neq(0,0)$, within the current $\mathrm{MB}$, calculate $F_{Q S}(u, v)=F^{\prime}(u, v) \times 16 / Q_{M}$, $\left(\left|I_{Q}(u, v)\right| \times Q_{S}\right)_{u p}$ and $\left(\left|I_{Q}(u, v)\right| \times Q_{S}\right)_{\text {down }}$.

2. Round $F_{Q S}(u, v)$ to the nearest even integer value $K(u, v)$.

3. Set all $K(u, v)$ less than 4 to zero (All DC values are set to $0)$.

4. Calculate the $Q_{S}$ upper bound $Q_{S}^{u p}$ by $\min \left(\left(I_{Q} \times Q_{S}\right)_{u p}\right)(\min$ is over the non-zero DCT coefficients).

5. For $j \in\left\{Q_{S}\right\}$ and $4 \leq j \leq Q_{S}^{u p}$, :

$$
\hat{Q}_{S}=\arg \max _{j}\left[N_{1}(j)+N_{2}(j)\right]
$$

where $N_{1}\left(Q_{S}\right)$ is the number of DCT coefficients for which $K(u, v)=Q_{S}$, and $N_{2}\left(Q_{S}\right)$ is the number of $K(u, v)$, which are divisible by $Q_{S}$.

6. For MBs, which do not contain any non-zero AC coefficients, the steps above do not provide a result. Instead, the estimated $\hat{Q}_{S}$ value from the previous MB is used for the current MB.

\subsection{Validation and I frame detection}

MPEG2 I frame detection based on the number of zero coefficients in each frame has been proposed [6]. Based on this number, an adaptive threshold filter was defined to determine an I frame threshold. Two limitations for this method are that it uses a preset value for the (approximate) GOP length and an initial value of the decision threshold.

Here a novel I frame detection method is proposed which makes decisions at frame level and therefore may be applied to adaptive GOP structures. A frame level mismatch measure $M_{F}$, is introduced to measure how accurate the step size is estimated,

$$
M_{F}=\sum_{M B} M_{M B}\left(\hat{Q}_{M}, \hat{Q}_{S}\right)
$$

Small values of $M_{F}$ (related to the distance between $F^{\prime}(u, v)$ and the closest multiple of the quantization step size) are obtained when the $Q_{S}$ values of the frame are correctly estimated. For I frames, where a very high percentage of the $Q_{S}$ values are correctly estimated, the mismatch, $M_{F}$, is mainly due to the rounding error $E_{r}$ and integer division shift $S$. For the other frame types, the motion compensated contributions will lead to many misleading contributions to the DCT coefficients which in turn leads to wrong quantization step size estimates. A threshold is applied to $M_{F}$.

\section{PSNR ESTIMATION}

The I frame peak signal to noise ratio (PSNR) can be estimated based on the predicted quantization step size values. In [3], no reference PSNR estimation was presented for I frames which are MPEG2 coded using a fixed $Q_{S}$. The value of $Q_{S}$ was estimated based on a frequency analysis of the DCT coefficients for each frequency, $(u, v)$. The distribution of the DCT coefficients was assumed to follow a Laplacian distribution prior to coding, but the parameter of the distribution $(\lambda)$ was estimated based on the DCT transform on the decoded video.

A method was given in [3] for calculating the overall mean square error DC distortion $\varepsilon_{D C}^{2}$ and $\mathrm{AC}$ distortion $\varepsilon_{A C}^{2} \cdot \varepsilon_{A C}^{2}$ was derived as:

$$
\varepsilon_{A C}^{2}=2 \lambda^{2}-\frac{2 \lambda \Delta e^{-\alpha / \lambda} e^{-\Delta / 2 \lambda}}{1-e^{-\Delta / \lambda}}\left[\frac{\alpha}{\lambda}+1\right]
$$

where $\Delta$ is the quantization step size as (2), and $\alpha$ is the shift factor in the MPEG-2 quantization scheme. $\lambda$ is the Laplacian parameter for each DCT coefficient. The value of $\Delta, \alpha$ and $\lambda$ can vary for different $\mathrm{AC}$ frequencies $(u, v)$, leading to a dependency on $Q_{M}$ and $Q_{S}$.

The average distortion $D$ at frame level was calculated by combining the estimated $Q_{S}$ and the coefficient distribution estimation.

$$
D=\frac{1}{64}\left[\varepsilon_{D C}^{2}+\sum_{i=1}^{63} \varepsilon_{A C}^{2}(i)\right]
$$

Usually sequences are coded using rate control, in which case $Q_{S}$ will vary from $\mathrm{MB}$ to $\mathrm{MB}$ within one frame. The distortion expression, (6) is here generalized to allow $Q_{S}$ to vary over MBs,

$\hat{D}=$

$\frac{\varepsilon_{D C}^{2} \times B_{v} \times B_{h}+\sum_{Q_{S}} \sum_{Q_{M}} \varepsilon_{A C}^{2}\left(Q_{S}, Q_{M}\right) \times N_{A C}\left(Q_{S}, Q_{M}\right)}{B_{v} \times B_{h}+\sum_{Q_{S}} \sum_{Q_{M}} N_{A C}\left(Q_{S}, Q_{M}\right)}$

where $B_{v}$ and $B_{h}$ are the number of DCT blocks vertically and horizontally and $N_{A C}\left(Q_{S}, Q_{M}\right)$ is the number of AC coefficients for the $\left(Q_{S}, Q_{M}\right)$ combination.

In [3] $\lambda$ was estimated for each DCT coefficient based on analysis of the second moment for each of the first $24 \mathrm{AC}$ coefficients (in zigzag scan order). Using the second moment will lead to overestimation of the parameter in case of outliers (or a heavy tail distribution.) Our guess is that this may have lead the authors to apply a different estimator (assuming $2 \lambda^{2}$ as the variance) for the high frequencies to counteract this effect.

Estimating $\lambda$ based on the number of zero coefficients provides a faster and simpler estimation, which readily matches the use of decoder side statistics. The idea was introduced in [7], and we derive the $\lambda$ estimate:

$$
\lambda\left(Q_{S}, Q_{M}\right)=-\frac{\Delta / 2+\alpha}{\ln \left(1-p_{0}\left(Q_{S}, Q_{M}\right)\right)}
$$

where $p_{0}\left(Q_{S}, Q_{M}\right)$ is the ratio of number of zero coefficients for all the coefficients quantized by $Q_{S}$ and $Q_{M}, p_{0}=N_{0} / N$, where $N_{0}$ is the estimated number of zero coefficients estimated by the number of recontructed values in the interval $[-\Delta / 2-\alpha, \Delta / 2+\alpha]$, and $N$ is the total number of coefficients. 


\section{RESULTS}

We used four SD progressive test sequences CITY, SOCCER (SOC), ICE and CREW having a resolution of $704 \times 576$. The sequences were coded at constant bitrates of $2 \mathrm{M}, 3 \mathrm{M}$ and $4 \mathrm{Mbits} / \mathrm{s}$ using MPEG2 default intra $Q_{M}$ and frame MB processing. The MPEG GOP length was $N=12$ and $2 \mathrm{~B}$-frames $(M=3)$ between P-frames were used in this test.

The MPEG2 parameters were extracted only using the decoded Y component. Two candidate intra $Q_{M}$ were used as mentioned previously. The correct $Q_{M}$ was identified for all frames. Since $Q_{S}$ can vary from $\mathrm{MB}$ to $\mathrm{MB}, \mathrm{MB}$ based $Q_{S}$ estimation was performed. For the 12 MPEG2 coded sequences (100 frames for each), very accurate estimates was achieved by the $Q_{S}$ algorithm (Table 1). The overall average true $Q_{S}$ value is 15.33 and the average estimated $Q_{S}$ value is 15.41

As a test, the decoded test sequences were also up-scaled to $1080 \mathrm{p}$, the block size was identified and thereafter down-scaled using cubic interpolation. The same MPEG2 analysis scheme was applied to these re-scaled sequences with different parameters. The rounding error $E_{r}$ range was adjusted to $\left[-2 E_{\max }, 2 E_{\max }\right]$. In $Q_{S}$ estimation algorithm Step 5), $j \geq 6$ instead of 4 . An accurate $Q_{S}$ estimation is also achieved in this case with an overall average $Q_{S}$ of 15.45 , demonstrating the robustness of the algorithm.

Table 1 shows the I frame PSNR estimation results using $\lambda$ values dependent on both $Q_{M}$ and $Q_{S}$. (CITY2 refers to CITY at 2M.) Based on the $Q_{S}$ estimation of Sect.3.2, method M1 adjusts $\varepsilon_{A C}^{2}$ (4) by the MB based $Q_{S}$ (instead of the average $Q_{S}$ ) and uses the new average distortion function in (6). For each $\left(Q_{S}, Q_{M}\right), \lambda$ is estimated as in [3]. Based on M1, M2 instead applies $\lambda\left(Q_{S}, Q_{M}\right)$ estimation based on the number of zero coefficients, by (7).

In Table 2, a weighted average over $\lambda\left(Q_{S}, Q_{M}\right)$ is used. The $\lambda_{a v g}$ is independent of $Q_{S}$ and only depends on $Q_{M}(u, v)$, i.e. AC positions $(u, v)$. Methods M3 and M4 are based on M1 and M2 respectively, but replacing $\lambda\left(Q_{S}, Q_{M}\right)$ by $\lambda_{a v g}$. In Tables 1 and 2, PSNR estimation results based on the actual quantization step size values are also given in parentheses for comparison. The average is calculated over all the I frames among the 100 frames of each sequence. It may be noted that the PSNR estimates using estimated and actual $Q_{S}$ values are virtually identical.

Figure 3 shows the I frame estimation results for the 12 coded sequences (100 frames for each sequence at $2 \mathrm{M}, 3 \mathrm{M}$ and $4 \mathrm{Mbits} / \mathrm{s}$ ) concatenated to one video stream. I frames are marked in (red) bars and the other frames are in green. The frames, which are assigned a wrong frame type are marked in (blue) squares. It is shown that $M_{F}$ gives a very clear and better separation of I frames than for the number of zero coefficients, thus also validating the analysis. The method proposed uses a mismatch threshold at 0.03 . Compared with our implementation of [6] based on zero coefficients, it improves the accuracy from $99.17 \%$ to $100 \%$.

Table 1. I frame, $Q_{S}$ and PSNR estimation results, $\left(\lambda\left(Q_{S}, Q_{M}\right)\right)$ \begin{tabular}{|l|c|c|c|c|c|c|c|c|c|c|c|c|c|}
\hline & CITY2 & SOC 2 & ICE 2 & CREW & CITY3 & SOC 3 & ICE 3 & CREW3 & CITY4 & SOC 4 & ICE 4 & CREW4 \\
\hline avg $Q_{S}$ & 24.70 & 29.29 & 11.11 & 21.53 & 16.67 & 18.02 & 7.50 & 12.79 & 13.12 & 13.90 & 6.24 & 10.00 \\
& $(24.70)$ & $(29.19)$ & $(11.21)$ & $(21.35)$ & $(16.67)$ & $(18.04)$ & $(7.34)$ & $(12.77)$ & $(13.12)$ & $(13.92)$ & $(5.69)$ & $(10.00)$ \\
\hline PSNR & 31.76 & 31.52 & 39.82 & 35.91 & 33.96 & 33.96 & 41.56 & 38.01 & 35.38 & 35.47 & 42.75 & 39.08 \\
\hline M1 & 31.35 & 30.52 & 38.25 & 33.26 & 34.59 & 34.19 & 41.48 & 37.12 & 36.62 & 36.31 & 43.12 & 39.11 \\
& $(31.36)$ & $(30.55)$ & $(38.26)$ & $(33.39)$ & $(34.59)$ & $(34.19)$ & $(41.63)$ & $(37.17)$ & $(36.62)$ & $(36.31)$ & $(43.78)$ & $(39.13)$ \\
\hline M2 & 31.88 & 32.09 & 37.95 & 35.32 & 33.95 & 34.28 & 40.07 & 37.57 & 35.24 & 35.68 & 41.19 & 38.81 \\
& $(31.88)$ & $(32.10)$ & $(37.95)$ & $(35.36)$ & $(33.95)$ & $(34.27)$ & $(40.17)$ & $(37.57)$ & $(35.34)$ & $(35.67)$ & $(41.66)$ & $(38.80)$ \\
\hline
\end{tabular}

\section{CONCLUSIONS}

Based on decoded MPEG2 video (without access to the MPEG stream), methods for estimating some coding parameters were pre-
Table 2. I frame, $Q_{S}$ and PSNR estimation results, $\left(\lambda_{\text {avg }}\right)$ \begin{tabular}{|l|c|c|c|c|c|c|c|c|c|c|c|c|}
\hline & CITY2 & SOC 2 & ICE 2 & CREW & CITY3 & SOC 3 & ICE 3 & CREW3 & CITY4 & SOC 4 & ICE 4 & CREW4 \\
\hline PSNR & 31.76 & 31.52 & 39.82 & 35.91 & 33.96 & 33.96 & 41.56 & 38.01 & 35.38 & 35.47 & 42.75 & 39.08 \\
\hline M3 & 31.50 & 30.72 & 38.54 & 33.63 & 34.71 & 34.34 & 41.89 & 37.40 & 36.72 & 36.43 & 43.96 & 39.34 \\
& $(31.50)$ & $(30.72)$ & $(38.53)$ & $(33.63)$ & $(34.71)$ & $(34.34)$ & $(41.90)$ & $(37.40)$ & $(36.72)$ & $(36.43)$ & $(43.99)$ & $(39.34)$ \\
\hline M4 & 31.87 & 32.07 & 38.38 & 35.07 & 34.08 & 34.42 & 40.88 & 37.79 & 35.54 & 35.83 & 42.20 & 39.21 \\
& $(31.88)$ & $(32.09)$ & $(38.33)$ & $(35.11)$ & $(34.08)$ & $(34.42)$ & $(40.91)$ & $(37.80)$ & $(35.54)$ & $(35.83)$ & $(42.40)$ & $(39.20)$ \\
\hline
\end{tabular}

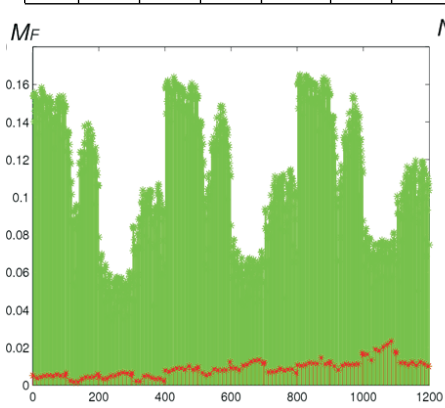
Navg

Fig. 3. I frame detection results for frames of the concatenated sequence. Left) By $M_{F}$ and Right) By the average number of zero coefficients per MB, $N_{\text {avg }}$.

sented. For I-frames, an algorithm to estimate the MB quantizer scale parameter was given. Tested on MPEG2 video with fixed bitrate and thereby varying $Q_{S}$, very good estimates were obtained. Combined with modeling and estimating the DCT coefficients distribution, good no reference estimates of the I frame PSNR was obtained. The difference of using the estimated and the actual $Q_{S}$ values was negligible. A novel I frame detector operating on individual frames based on evaluating the validity of the I frame $Q_{S}$ estimation was also presented, showing a clear separation of the I frames from the motion compensated frames.

\section{ACKNOWLEDGMENT}

We would like to thank Jesper Meldgaard Pedersen of the Display \& Picture group at Bang \& Olufsen for fruitful discussions.

\section{REFERENCES}

[1] A.lchigaya, M.Kurozumi, N.Hara, Y.Nishida, and E.Nakasu, "A method of estimating coding PSNR using quantized DCT coefficients," IEEE Trans. Circ. Syst Video Tech., vol. 16, pp. 251259, Feb. 2006.

[2] C.Kim, "Adaptive post-fltering for reducing blocking and ringing artifacts in low bit-rate video coding," Signal Processing: Image Communication., vol. 17, pp. 525-535, Apr. 2002.

[3] D.S.Turaga, Y.Chen, and J.Caviedes, "No reference PSNR estimation for compressed pictures," Signal Processing: Image Communication, vol. 19, pp. 173-184, Sep. 2004.

[4] "MPEG2 Video Standard, Information Technology-Generic Coding of Moving Pictures and Associated Audio information:Video, ISO/IEC 13818-2,” 2000.

[5] W.Fischer, "Digital television, a practical guide for engineers," Springer-Verlag Berling Heidelberg, 2004.

[6] M.J.Knee and I.J.Poole, "Analysis of compression decoded video image sequences," United States Patent, US 6895049 B1, May 2005.

[7] Z.He and S.Mitra, "Rate-distortion modeling for efficient h.264/AVC encoding," IEEE Trans. Circ. Syst Video Tech., vol. 11, pp. 1221-1236, Dec. 2001. 